\title{
MULTISCALE COUPLINGS IN PROTOTYPE HYBRID DETERMINISTIC/STOCHASTIC SYSTEMS: PART II, STOCHASTIC CLOSURES*
}

\author{
M. A. KATSOULAKIS ${ }^{\dagger}$, A. J. MAJDA ${ }^{\ddagger}$, AND A. SOPASAKIS ${ }^{\S}$
}

\begin{abstract}
Couplings of microscopic stochastic models to deterministic macroscopic ordinary and partial differential equations are commonplace in numerous applications such as catalysis, deposition processes, polymeric flows, biological networks and parametrizations of tropical and open ocean convection. In this paper we continue our study of the class of prototype hybrid systems presented in [8]. These model systems are comprised of a microscopic Arrhenius dynamics stochastic process modeling adsorption/desorption of interacting particles which is coupled to an ordinary differential equation exhibiting a variety of bifurcation profiles. Here we focus on the case where phase transitions do not occur in the microscopic stochastic system and examine the influence of noise in the overall system dynamics.

Deterministic mean field and stochastic averaging closures derived in [8] are valid under stringent conditions on the range of microscopic interactions and time-scale separation respectively. Furthermore, their derivation is valid only for finite time intervals where rare events will not trigger a large deviation from the average behavior in the zero noise limit. In this paper we study such questions in the context of simple hybrid systems, demonstrating that deterministic closures based on various separation of scales arguments cannot in general capture transient and long-time dynamics. For this purpose we develop coarse grained stochastic closures for this class of hybrid systems and compare them to deterministic, mean-field and stochastic averaging closures. We show that the proposed coarse grained closures describe correctly the microscopic hybrid system solutions in all test cases examined, including rare events and random transitions between multiple stable states.
\end{abstract}

Key words. Coupled Hybrid Systems; Stochastic Closures; Multi Scale Interactions; Critical Phenomena; Monte Carlo Methods.

AMS subject classifications. 34E $13 ; 82 \mathrm{C} 27 ; 93 \mathrm{C} 15 ; 37 \mathrm{M} 20 ; 65 \mathrm{C} 05$;

\section{Introduction}

In this paper we study the influence of micro-/sub- grid scale fluctuations in hybrid dynamical systems consisting of coupled macroscopic, deterministic differential equations and microscopic, stochastic lattice particle systems. We focus on model prototype systems proposed in the earlier companion paper [8] that are computationally tractable with direct numerical simulation even for long times, but still can exhibit a host of complex phenomena, as further demonstrated here. Our findings strongly suggest that deterministic closures of the hybrid system such as mean field, stochastic averaging or moment equations may be either inadequate as descriptions of the overall system or simply difficult to both derive and assess in their effectiveness. Motivated by such considerations, we propose a class of stochastic closures based on systematic coarse-graining of the microscopic stochastic lattice dynamics that yield computationally inexpensive reduced hybrid models and capture correctly the transient and long-time behavior of the full hybrid system.

Couplings of atomistic or molecular, and more generally microscopic stochastic models to deterministic macroscopic ordinary and partial differential equations (ODE/PDE) are commonplace in applications ranging from catalysis and deposition

\footnotetext{
${ }^{*}$ Received: June 13, 2005; accepted (in revised version): July 15, 2005. Communicated by Shi Jin.

${ }^{\dagger}$ Department of Mathematics and Statistics, University of Massachusetts, Amherst, MA 01003.

${ }^{\ddagger}$ Courant Institute of Mathematical Sciences and Center for Atmospheric and Ocean Sciences, New York University, New York, NY 10012.

$\S^{\S}$ Department of Mathematics and Statistics, University of Massachusetts, Amherst, MA 01003.
} 
processes to polymeric flows, to stochastic models for tropical and open ocean convection and complex biological networks, see for instance [20, 19, 23, 17, 21, 24]. In this category of problems microscopic stochastic processes, typically simulated by Monte Carlo (MC) methods, model small scale activity, for instance adsorption, desorption, surface reaction and surface diffusion of particles on an interface or boundary layer, in contact with a gas/fluid phase. The micromechanisms are interrelated with the large scale fluid flow modeled by continuum ODE/PDE describing the evolution of fluid and thermodynamic variables.

Challenges in all these hybrid problems arise in the direct numerical simulation of realistic size systems due to scale disparities between the discrete stochastic microscopic models and the continuum macroscopic equations; secondly, the fact that the coupled systems have nonlinear interactions across a wide range of scales, implies that the stochasticity inherited from the microscopic model can play a subtle but important role in the dynamic behavior of the overall system. In this paper we address such issues in the context of prototype mathematical models of a deterministic ODE coupled with a stochastic spin flip/spin exchange Ising model, that capture essential features of complex hybrid systems. These models were first proposed in [8] where we obtained deterministic mesoscopic models from the hybrid system by employing two methods: stochastic averaging principle and mean field closures, focusing on the case where phase transitions do not occur in the stochastic system. In the averaging principle case a faster stochastic mechanism was assumed compared to the ODE relaxation; as a result, local equilibrium - parametrized with respect to the slow, deterministic variables - was enforced with respect to the Gibbs measure on the lattice system. Under these circumstances remarkable agreement was numerically observed between the hybrid system and the averaged system predictions. In fact, one of the principal motivations behind introducing the model systems in [8] was specifically to allow for testing the derivation of computationally inexpensive mesoscopic closures for the average behavior of the hybrid systems in various asymptotic limits, and to have computationally feasible detailed comparisons of the derived mesoscopic theories against direct numerical simulations of the full hybrid system.

In this paper we specifically examine the influence of micro-/sub- grid scale noise in hybrid dynamical systems. Although in [8] remarkable agreement was observed between direct numerical simulations of hybrid systems and deterministic closures, long-time simulations and asymptotic analysis in a linearized stochastic PDE limit strongly suggest that fluctuation-driven rare events do occur in several parameter regimes and are not captured by the deterministic mesoscopic equations; in fact this possibility can be mathematically quantified in the case of stochastic averaging for systems of stochastic differential equations using large deviations arguments, [6]. Similar issues may also arise when strong noise is present in the system, in which case fluctuations play a significant role in the overall dynamics; for instance noise is important when the external deterministic system exhibits multiple steady states (e.g. bistability, phase transitions, etc.) or when bifurcations of various types may arise. In this paper we demonstrate such phenomena in the context of simple hybrid systems and further demonstrate that deterministic closures based on various separation of scales arguments cannot capture transient and long-time dynamics. This profound influence of noise is missed by the aforementioned methods, in spite of the fact that they may rely on many occasions on rigorous derivations: all theoretical results are derived on finite-time intervals, where the influence of noise asymptotically vanishes due to the Law of Large Numbers scalings that are typically employed. We refer to 
the relevant discussion in Section 4 of this paper. Similarly, computational closure methods relying on relatively short runs of microsimulators such as the EquationFree, [14] and HMM [4] approaches may also have to account for such phenomena when deciding the integration time for the micro simulation and determining suitable coarse-grained observables such as a number of moments.

As a result of these observations which are further detailed in Section 4, it is evident that we need to develop mathematical strategies that allow fluctuations to be properly and systematically included in the deterministic mesoscopic models. In this paper, we address this issue by employing recently developed coarse-grained stochastic models for lattice dynamics of Ising-type systems [9, 10, 13], in order to describe the microscopic mechanisms with a hierarchy of coarser (and thus computationally preferable) but still discrete, stochastic observables. The proposed class of stochastic closures, presented in Section 5, indeed gives rise to computationally inexpensive reduced hybrid models that capture correctly the transient and long-time behavior of the full hybrid system. In particular, as demonstrated with detailed comparisons to direct numerical simulations in Section 6, the coarse-grained stochastic closures yield pathwise agreement with the full hybrid system even in regimes where rare events may occur and through random transitions in multiple steady states and bifurcation regimes. We also refer to [3] regarding multiscale methods for Ising-type systems which are based on renormalization group and multigrid ideas. Finally we remark that a hybrid coarse-grained model along the lines of the ones studied here, was already introduced in [15] as a stochastic parameterization of unresolved features of tropical convection; such models are expected to provide a computationally efficient stochastic model whose predictions can be directly related to observational data.

In this paper we focus on hybrid systems where phase transitions do not occur in the microscopic lattice dynamics, but rather complex dynamic behavior is primarily due to the bifurcations of the externally coupled deterministic system, and its coupling with the microscopic stochastic model. Phase transitions in the microscopic lattice system are due to strong particle/particle interactions and such hybrid systems demonstrate even more complex behavior than the ones considered here, for instance regimes of strong intermittency, and random oscillations; this case is treated separately in a follow-up publication.

\section{Hybrid models}

We introduce the microscopic spin flip stochastic Ising process $\left\{\sigma_{t}\right\}_{t \geq 0}$, modeling the adsorption and desorption of particles on a one-dimensional surface, coupled to an ODE that serves as a caricature of an overlying gas-phase dynamic; we also refer to [23] for such a system arising in the modeling of well-mixed reactors in catalysis.

The microscopic stochastic process is defined by its generator $L$ and the two-way coupled system is written as

$$
\begin{aligned}
\frac{d}{d t} \vec{X} & =\frac{1}{\tau_{c}} G(\vec{X}, \bar{\sigma}) \\
\frac{d}{d t} E f(\sigma) & =E L f(\sigma)
\end{aligned}
$$

where $\bar{\sigma}$ denotes a spatial average coverage, $f$ is a test function, $G$ the ODE function and $\tau_{c}$ corresponds to the characteristic time for the ODE. We provide all further details for system $(2.1,2.2)$ below. Examples of ODEs such as $(2.1)$ considered here are scalar equations with bistable behavior or saddle node bifurcations, as well as a 
spatially homogeneous complex Ginsburg-Landau equation exhibiting Hopf bifurcations. In addition to the spin flip mechanism, we can also consider spin exchange, as well as combined spin flip / spin exchange mechanisms. In this paper we concentrate only on one of them, namely spin flips.

We start with a detailed description of (2.2). We consider a microscopic stochastic model defined on a periodic lattice of size $N$ which we denote by $\mathcal{L}=\{1,2, \ldots, N\}$. At each lattice site $x \in \mathcal{L}$, an order parameter $\sigma$, is allowed to take the values 0 or 1. In accordance with the classical Ising model we refer to the order parameter as spin. We will assume that sites cannot be occupied by more than one particle. A spin configuration $\sigma$ is an element of the configuration space $\Sigma=\{0,1\}^{\mathcal{L}}$ and we write $\sigma=\{\sigma(x): x \in \mathcal{L}\}$ denoting by $\sigma(x)$ the spin at $x$. Physically this mechanism may describe the desorption of a particle from a surface described by the lattice to the gas phase above and conversely the adsorption of a particle from the gas phase to the surface. Similarly it can describe phase transitions without order parameter conservation although here we concentrate on the case where phase transitions are absent in the microscopic system.

The stochastic process $\left\{\sigma_{t}\right\}_{t \geq 0}$ is a continuous time jump Markov process on $L^{\infty}(\Sigma, R)$ with generator, [16],

$$
L f(\sigma)=\sum_{x \in \mathcal{L}} c(x, \sigma)\left[f\left(\sigma^{x}\right)-f(\sigma)\right]
$$

for any test function $f \in L^{\infty}(\Sigma, R)$. Here $\sigma^{x}$ signifies the configuration after a flip at $x$,

$$
\sigma^{x}(y)=\left\{\begin{array}{cc}
1-\sigma(x), & \text { if } y=x \\
\sigma(y), & \text { if } y \neq x
\end{array}\right.
$$

and $c(x, \sigma)$ denotes the rate of a spin flip at $x$ for the configuration $\sigma$ (see 2.6).

Since there are $N=|\mathcal{L}|$ sites on the lattice then the system can be in any of $2^{|\mathcal{L}|}$ possible states while the energy of any particular state is given by the following Hamiltonian,

$$
H(\sigma)=-\frac{1}{2} \sum_{x \in \mathcal{L}} \sum_{y \neq x} J(x, y) \sigma(x) \sigma(y)+\sum_{x} h \sigma(x)
$$

where $J(x, y)$ is an inter-particle potential and $h$ is a given external potential. We let,

$$
J(x, y)=\frac{1}{2 L+1} J\left(\frac{|x-y|}{2 L+1}\right)
$$

where $L$ denotes the range of microscopic interactions. Here $J$ is assumed to be even, $J(r)=J(-r)$ and as an example, can take a form similar to [9],

$$
J(r)= \begin{cases}J_{0} & \text { if } 0 \leq r \leq 1 \\ 0 & \text { otherwise }\end{cases}
$$

where $J_{0}$ is a parameter which based on its sign describes attractive, repulsive or no-interactions. For attractive microscopic interactions for instance, $J_{0}$ is positive.

By applying a spin flip stochastic model we create new states from old ones generated by a Markov process as described below. Equilibrium states of the stochastic 
model are described by the Gibbs states at the prescribed temperature $T$. If we denote the inverse temperature by $\beta=1 /(k T)$ we then have,

$$
\mu_{\beta, N}(d \sigma)=\frac{1}{Z} e^{-\beta H(\sigma)} P_{N}(d \sigma)
$$

where $P_{N}(d \sigma)$ denotes the (product) prior distribution on $\mathcal{L}$,

$$
P_{N}(d \sigma)=\prod_{x \in \mathcal{L}} \rho(d \sigma(x)) \text { and } \rho(\sigma(x)=0)=\frac{1}{2}, \rho(\sigma(x)=1)=\frac{1}{2} .
$$

Here $Z$ is the partition function, guaranteeing that (2.5) is a probability measure.

In this model we implement spin flip Arrhenius dynamics. Under this type of mechanism the simulation is driven based on the energy barrier a particle has to overcome in flipping from one state to another given be (2.7) below. For Metropolis and other choices of dynamics see [9]. The Arrhenius spin flip rate $c(x, \sigma)$ at lattice site $x$ and spin configuration $\sigma$ is given by

$$
c(x, \sigma)= \begin{cases}c_{d} e^{-\beta\left[U_{0}+U(x)\right]}, & \text { when } \sigma(x)=1, \\ c_{a} & \text { when } \sigma(x)=0,\end{cases}
$$

where,

$$
U(x)=\sum_{\substack{z \neq x \\ z \in \mathcal{L}}} J(x, z) \sigma(z)-h
$$

with absorption / desorption constants,

$$
c_{a}=c_{d}=1 / \tau_{I}
$$

and $\tau_{I}$ denotes the characteristic time of the stochastic process. Here $U_{0}$ represents the energy associated with the surface binding of the particle at location $x$ which we set to $U_{0}=0$.

In general we write the spin flip rate (2.6) as,

$$
c(x, \sigma):=c_{a}(x, \sigma)+c_{d}(x, \sigma)=c_{a}(1-\sigma(x))+c_{d} \sigma(x) \exp \left(-\beta\left(U_{0}+U(x)\right)\right.
$$

and therefore the probability of a spin flip at $x$ during time $[t, t+\Delta t]$ is,

$$
c(x, \sigma) \Delta t+O\left(\Delta t^{2}\right) .
$$

The dynamics as described here leave the Gibbs measure (2.5) invariant, since they satisfy the detailed balance condition

$$
c(x, \sigma)=c\left(x, \sigma^{x}\right) \exp \left(-\beta \Delta_{x} H(\sigma)\right)
$$

where

$$
\Delta_{x} H(\sigma)=H\left(\sigma^{x}\right)-H(\sigma) .
$$

We present below some of the candidate examples for our ODE (2.1). The formulations studied were chosen in order to display interesting solution profiles through succinct and simple equations. They include bistable, saddle and Complex Ginzburg 
Landau (CGL) equations [1]. The ODE (2.1) and the stochastic system (2.2) are coupled via, respectively, the external field, $h \equiv h(\vec{X})=c X+h_{0}$ with $\vec{X}=X+Y i$ for appropriate constants $c$ and $h_{0}$ whose values are specified for each case examined and the area fraction (or total coverage) defined as the spatial average of the stochastic process $\sigma, \bar{\sigma}=\frac{1}{N} \sum_{x \in \mathcal{L}} \sigma(x)$.We summarize here the main types of examples and stability behavior which we will study in more detail later. All parameters involved in the formulations below are explicitly provided in the simulation Section 6.2. Here $\tau_{c}$ denotes the characteristic time for the ODE.

- Example 1 (Bistable):

$$
G(X, \sigma)=a(\bar{\sigma}) X+\tilde{\gamma} X^{3}, \text { where } a(\bar{\sigma})=b(1-\bar{\sigma})+z \quad \tilde{\gamma}<0
$$

In particular bistability arises according to the sign of $a(\bar{\sigma})$. If $a(\bar{\sigma}) \leq 0$ we have a stable node at 0 while otherwise we have two stable nodes at $\pm \sqrt{-a(\bar{\sigma}) / \tilde{\gamma}}$.

- Example 2 (Saddle):

$$
G(X, \sigma)=a(\bar{\sigma})+\tilde{\gamma} X^{2}, \text { where } a(\bar{\sigma})=b(z-\bar{\sigma}) .
$$

Similarly this example displays saddle behavior depending on the sign of $a(\bar{\sigma}) / \tilde{\gamma}$. For $a(\bar{\sigma}) / \tilde{\gamma}>0$ there exist no nodes while otherwise we obtain one stable and one unstable node at $\pm \sqrt{-a(\bar{\sigma}) / \tilde{\gamma}}$.

- Example 3 (CGL):

$$
G(\vec{X}, \sigma)=\left[\left(\begin{array}{cc}
a(\bar{\sigma})+\gamma & -\omega \\
\omega & a(\bar{\sigma})-\gamma
\end{array}\right)-\tilde{\gamma}|\vec{X}|^{2}\right] \vec{X}, \text { where: } \begin{aligned}
& a(\bar{\sigma})=b(\bar{\sigma}-z), \\
& \vec{X}=X+Y i
\end{aligned}
$$

This example exhibits a Hopf bifurcation. The Jacobian of the linearized system has eigenvalues

$$
\lambda=\lambda(\bar{\sigma})=a(\bar{\sigma}) \pm i \sqrt{\omega^{2}-\gamma^{2}}
$$

and in general we have either a stable node at $(0,0)$ for $a(\bar{\sigma})<0$ or a limit cycle otherwise.

The parameters in each example are chosen so that the noise constantly probes the critical values for which bifurcations occur under each of the examples above. The coupled system therefore can move between regions of stability or instability based on the coupling noise parameter $\bar{\sigma}$. Further details on the exact values of all parameters used and respective behavior of the bifurcations are to be found in the numerical simulations Section 6.2.

Each of the bifurcation ODEs $(2.10$ - 2.12) that enters in system $(2.1,2.2)$ has its own time scale, $\tau_{c}$ and competes with the characteristic time for the stochastic model $\tau_{I}$. We define

$$
\tau=\tau_{c} / \tau_{I}
$$

and note the following three possibilities:

- $\tau>>1 \Longrightarrow$ ODE equilibrates slower than spin flip model.

- $\tau \approx 1 \Longrightarrow$ ODE equilibrates at similar times as the spin flip model.

- $\tau<<1 \Longrightarrow$ ODE equilibrates faster than spin flip model,

where without loss of generality we fix $\tau_{I} \equiv 1$ and vary $\tau_{c}$ accordingly for the cases specified above. 
We will monitor in our simulations how the phase portrait of our dynamical system $(2.1,2.2)$ transforms when the critical parameter coupled to $\bar{\sigma}$ passes through its bifurcating value. We perform these numerical comparisons under the three cases of $\tau_{c}$ outlined above and a variety of values for the fixed parameters of the external potential and the ODEs corresponding to a wealth of different phase space portraits.

\section{Deterministic Closures and the Role of Stochastic Fluctuations}

In this section we outline deterministic closures of the hybrid system (2.2), (2.1) studied in $[8]$ in two distinct asymptotic regimes. First, in the case of weak long range interactions $L \rightarrow \infty$, we obtain a coupled, possibly spatially distributed mean field deterministic mesoscopic system for the evolution of $X$ and the average local coverage corresponding to the stochastic order parameter $\sigma$. Second, using stochastic averaging, we obtain an effective ODE for the variable $X=X(t)$ in the time-asymptotic limit $\tau>>1$, i.e. when the relaxation time $\tau_{I}$ of the stochastic system (2.2) is much faster than the relaxation time $\tau_{c}$ of the ODE (2.1). The rigorous derivations for these closures can be found in [8].

3.1. Local and Global Mean Field Models. In this subsection we obtain in the asymptotic limit of long range interactions $N=2 L+1$ for $N, L \rightarrow \infty$ a spatially distributed deterministic closure of (2.2), (2.1), without any time-scales separation assumption. In that case, equilibrium mean-field theory becomes asymptotically exact (see [8]). We briefly outline here the derivation of mean-field equations for our coupled system (2.2), (2.1). We consider the spin flip Arrhenius type dynamics which we presented in Section 2, and using the definition of the generator, we obtain the following time evolution law for the average coverage $E \sigma(x)$ and the average position $E \vec{X}$ :

$$
\left\{\begin{aligned}
\frac{d}{d t} E \vec{X} & =\frac{1}{\tau_{c}} E G(\vec{X}, \bar{\sigma}) \\
\frac{d}{d t} E \sigma(x) & =\frac{1}{\tau_{I}} E\left[(1-\sigma(x))-\sigma(x) e^{-\beta U(x ; \vec{X}, \sigma)}\right]
\end{aligned}\right.
$$

where $U(x ; \vec{X}, \sigma)$,

$$
U(x ; \vec{X}, \sigma)=\sum_{z \neq x} J(x, z) \sigma(z)-h(\vec{X}) .
$$

Note that (3.1) is exact but not closed in terms of $E \sigma(x)$ and $E X$. Although such a system cannot provide a predictive tool by itself, it can certainly be used, since it is exact, as a numerical benchmark for the deterministic and stochastic closures presented next.

In order to proceed further and close the system above we consider an averaged but still stochastic quantity, namely the empirical measure,

$$
\mu^{N}(d y, t)=\frac{1}{N} \sum_{x \in \mathcal{L}} \sigma_{t}(x) \delta_{x}(d y), \text { for } y \in R .
$$

Note that if $A \subset R$ then

$$
\mu^{N}(A, t)=\frac{\text { number of particles in } A}{N} .
$$

In this case our ODE, (2.1) becomes exactly,

$$
\frac{d \vec{X}}{d t}=G\left(\vec{X}, \bar{\mu}^{N}\right) \text { with } \bar{\mu}^{N}(t)=\int_{R} \mu^{N}(d z, t)=\bar{\sigma}_{t} .
$$


Passing to the weak-* limit in $\mu^{N}(d y, t)$ we have the following result: for a smooth long-ranged potential $J(x, y)=\frac{1}{N} J\left(\frac{|x-y|}{N}\right)$ and any finite time $T$, we have that

$$
\lim _{N \rightarrow \infty} X(t)=Y(t) \text { and } \lim _{N \rightarrow \infty} \mu^{N}(d y, t)=u(y, t) d y \text { uniformly in }[0, T]
$$

where $(Y, u)$ solves

$$
\left\{\begin{array}{l}
\frac{d}{d t} \vec{Y}=\frac{1}{\tau_{c}} G(\vec{Y}, \bar{u}) \\
\frac{d}{d t} u=\frac{1}{\tau_{I}}\left\{1-u-u e^{-\beta(J * u-h(\vec{Y}))}\right\}
\end{array} \quad \text { for } t \in[0, T], y \in[0,1]\right.
$$

$\bar{u}(t)=\int_{0}^{1} u(y, t) d y$ and $G$ corresponds to the given ODE as in (2.10), (2.11) or (2.12). As discussed in [8] relation (3.5) is analogous to the Law of Large Numbers yielding the mean behavior of the hybrid system and treating fluctuations as a higher order correction. Examples of different choices for $G$ will be considered in the numerical test cases below.

A particular but important sub-case follows here if we make the assumption that $J \equiv J_{0}$ i.e assuming a uniform interaction potential. We subsequently obtain the following mean field model ODE system describing total spatial averages,

$$
\left\{\begin{array}{l}
\frac{d}{d t} \vec{Y}=\frac{1}{\tau_{c}} G(\vec{Y}, \bar{u}) \\
\frac{d \bar{u}}{d t}=\frac{1}{\tau_{I}}(1-\bar{u})-\frac{1}{\tau_{I}} \bar{u} e^{-\beta J_{0} \bar{u}+\beta h(\vec{Y})} \quad \text { for } t \in[0, T] .
\end{array}\right.
$$

The exact mean-field formulations corresponding to each of our examples will be presented in the simulations Section 6.2 below.

3.2. Stochastic Averaging. We now assume that $\tau>>1$ which implies that the stochastic system evolves with a characteristic time which is much faster compared to that of the ODE. In this case we can apply a stochastic averaging principle, which is conceptually similar to the one for systems of stochastic differential equations with fast and slow scales, see for instance [6].

The main requirement here is the ergodicity property of the stochastic process $\left\{\sigma_{t}\right\}_{t \geq 0}$. Indeed, for any fixed, finite $N$ and $\vec{X}$, the process $\sigma$ is ergodic with (2.5) as the unique invariant measure, [18]; in particular we have,

$$
\lim _{T \rightarrow \infty} \frac{1}{T} \int_{0}^{T} G\left(\vec{X}, \bar{\sigma}_{t}\right) d s=\bar{G}(\vec{X}) \text { for } \vec{X} \in R^{2}
$$

where

$$
\bar{G}(\vec{X})=E_{\mu_{\beta, N}} G(\vec{X}, \bar{\sigma}) \text { for } \vec{X} \in R^{2} .
$$

Due to special structure of $G$ which depends on $\bar{\sigma}$ linearly (see $(2.10,2.11,2.12)$ ), we always have

$$
E_{\mu_{\beta, N}} G(\vec{X}, \bar{\sigma})=G\left(\vec{X}, u_{\beta, N}(h(\vec{X}))\right)
$$

where,

$$
u_{\beta, N}(h)=E_{\mu_{\beta, N}} \bar{\sigma}=\frac{1}{Z} \sum_{\{\sigma\}} \sum_{x \in \mathcal{L}} \sigma(x) e^{-\beta H(\sigma)} P_{N}(\sigma) .
$$


Furthermore (3.10) is the average coverage of the microscopic system which in equilibrium statistical mechanics is known as average magnetization. It is clear from the formula that for finite $N, u_{\beta, N}(h)$ is an analytic function of $h$, we refer to [5] (Theorem V.4.3) for a detailed discussion of its properties (e.g. monotonically increasing, concave for $h>0$, symmetry in $h$, etc.). In other words there are no first-order phase transitions for finite $N$, and in this case (3.9) and the averaged ODE (3.12) below are always well defined, regardless of temperature and strength of interactions. In fact we can show the following: for an arbitrary bounded time interval $[0, T]$ with fixed $N$ and $\tau_{c}$ we have

$$
\lim _{\tau_{I} \rightarrow 0} P\left(\sup _{0 \leq t \leq T}|\vec{X}(t)-\bar{x}(t)|>\delta\right)=0
$$

for any $\delta>0$ where $\vec{X}=\vec{X}(t)$ solves $(2.1)$, and $\bar{x}=\bar{x}(t)$ is the solution of the averaged system (depending on the lattice size $N$ )

$$
\left\{\begin{array}{l}
\frac{d}{d t} \bar{x}_{t}=\frac{1}{\tau_{c}} \bar{G}\left(\bar{x}_{t}\right) \\
\bar{G}\left(\bar{x}_{t}\right)=G\left(\bar{x}_{t}, u_{\beta, N}\left(h\left(\bar{x}_{t}\right)\right)\right) \quad \text { for } t \in[0, T] . \\
\bar{x}_{0}=x
\end{array}\right.
$$

REMARK 3.1. As pointed out earlier, for a finite $N$, the spin flip system has no phase transitions which can arise depending on the competition between fluctuations and interactions, in the $N \rightarrow \infty$ limit. Therefore the results above always apply since in that context $N$ is kept fixed, while $\tau_{I} \rightarrow 0\left(\tau=\tau_{c} / \tau_{I} \rightarrow \infty\right)$. However, even for finite $N$, phase transitions are manifested as hysteresis in Monte Carlo simulations (see for instance Figures 1 and 2 in [9]). A similar phenomenon arises also here, since the coupling of (2.1) to the spin flip system is through the external field $h=h(\vec{X})$. The phase transition case in the stochastic lattice system will be examined extensively in [7]; in this case the coupled system (2.1, 2.2) not only demonstrates metastability but also oscillations and intermittency. For practical ways which allow us to obtain and implement the averaged system (3.12) we refer to [8]. Once $u_{\beta, N}(h)$ has been determined it is an easy task to obtain the corresponding averaging closure for each of our ODE examples.

\section{Regimes of Validity of Deterministic Closures}

Both of the closures presented in the previous section have been successful under extensive numerical tests in [8] when applied reasonably within the limits of their respective underlying assumptions as outlined in the previous section. In this section we examine the impact of applying these closures in situations for which the underlying mathematical assumptions are expected to be violated and noise could be important.

One important underlying assumption for both the stochastic averaging and mean field closures is that they are valid for a finite time interval $[0, T],[8]$. In the case of infinite time intervals, even for small noise, large deviation effects may be present which can alter the stability of the hybrid system. Similarly if the coupling is subjected to high noise levels, (e.g. $\tau<<1$ ) deterministic closures are expected to fail. Alternatively changes in the system's temperature can create phase transitions, which in turn can generate a whole new set of phenomena (e.g. intermittency or oscillations) which do not exist in the uncoupled spin flip system. The results presented here deal with the case of absence of phase transitions in the spin flip system; the phase transition case is treated in detail in [7]. In all of the cases outlined above, the hybrid system may display transient and long-time behavior which can be completely different from 

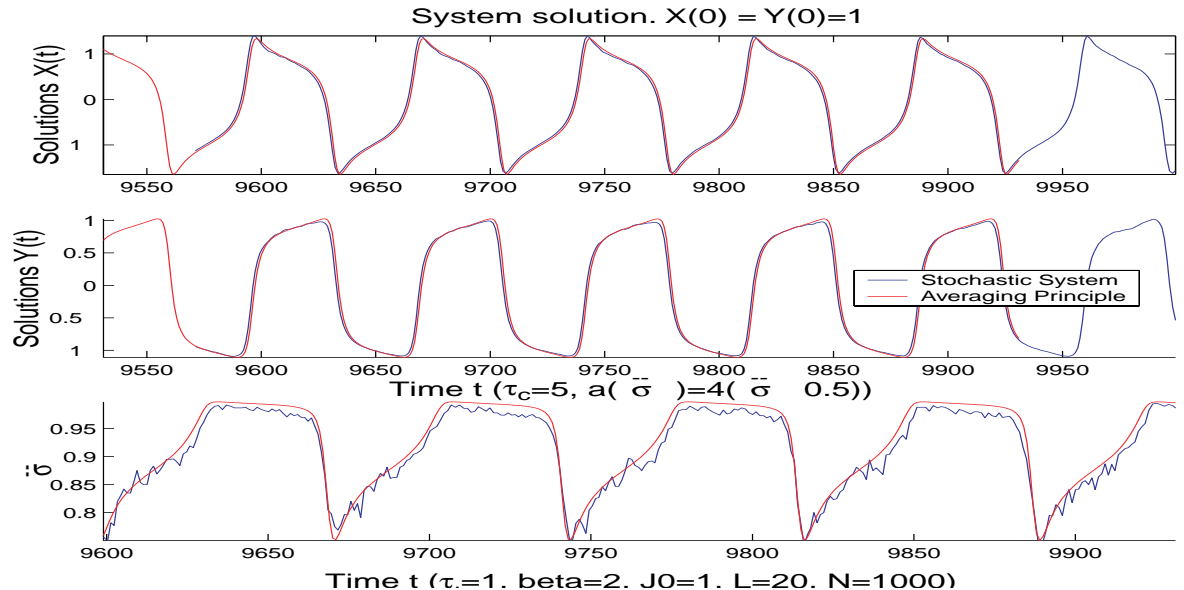

FIG. 4.1. Typical example of success for the stochastic averaging closure in the regime $\tau>>1$ for (2.12). Comparisons of solutions in time for the microscopic coupled system versus the stochastic averaging closure. See [8] for a discussion and details of the solutions. Parameters $\tau=5$ and $\beta J_{0}=2$.

those predicted by the deterministic closures. Chosen examples presented in this section display the capabilities as well as the shortcomings of deterministic closures. For instance, a case where stochastic fluctuations may be critical is the blow-up (solutions tending to $\infty$ ) examples which are studied in Section 4.2. In this case the analogue of (3.12) cannot predict blow-up which occurs for the direct numerical simulations of the full stochastic model.

Before we start examining the limitations for the deterministic closures presented in Section 3 we should briefly present a characteristic case where the approximation works well within the limits of validity of the underlying assumptions. As a typical test case of success for the stochastic averaging closure (3.12) we present a solution of our coupled system $(2.1,2.2)$ for the Hopf bifurcation ODE (2.12) in Figure 4.1 for $\tau>>1$. We therefore solve our hybrid model under the assumption of fast $(\tau=5)$ stochastic dynamics and in this case for $\beta J_{0}=2$. Note that here we are away from the phase transition regime which occurs for $\beta J_{0}=4$. It should be pointed out that there is a small but systematic error which is evident in our solution in Figure 4.1 due to the fact that we are approximating $u_{\beta}(h)$ via mean field theory [8]. Although the case $\tau=5$ is not much bigger than 1 as required by the mathematical theory (3.11), this stochastic averaging type closure performs well and approximates the solutions for coupled systems with fixed stable equilibria or single periodic orbit as long as we stay within the assumptions of its derivation. Several more such examples have been undertaken under different types of bifurcation couplings and a small fraction have been presented in [8].

We next examine three examples where stochastic fluctuations will be important. Under these circumstances we will present cases for which deterministic models are expected to fail.

4.1. Example 1: Bistable Case.

As a first such example we present a coupled system consisting of a bistable type bifurcation ODE with a shallow, double well potential (2.10). Our objective is to allow the stochastic process to induce 
spontaneous jumps of the hybrid system $(2.1,2.2)$ solution within a reasonable computation time; clearly deterministic closures such as mean field or stochastic averaging could not possibly capture such a solution correctly. Furthermore we could not apply stochastic averaging in this case since it would not be valid for relaxation times which here are as small as $\tau=.001$. In fact based on our results in [8] we know that for such relaxation times the stochastic noise is substantial thus inducing jumps of the solution between the potential wells. Although we do not examine the phase transition case in the spin flip system, here the behavior seen in our simulations is driven by phase transitions through the coupling in (2.2) with the external ODE.

More specifically we examine the coupled system (2.2), (2.1) with the following type of bistable bifurcating ODE,

$$
\frac{d X}{d t}=\frac{1}{\tau_{c}}\left(a(\bar{\sigma}) X+\tilde{\gamma} X^{3}\right),
$$

where we let $a(\bar{\sigma})=z+b(1-\bar{\sigma})$, with $b=4, z=-1$ and $\tilde{\gamma}=-100$. The external potential we apply in this example is linear and has the form, $h=h(X)=c X+h_{0}$ with $c=-1, h_{0}=.005$. Note that as long as $a(\bar{\sigma})>0,(4.1)$ has three nodes: $0, \pm \sqrt{-a(\bar{\sigma}) / \tilde{\gamma}}$; furthermore (4.1) has two main bifurcation states: bistable at $\pm \sqrt{-a(\bar{\sigma}) / \tilde{\gamma}}$ or single node at 0 .

The mean-field formulation and stochastic averaging closure corresponding to the top equation of (3.7) for this bistable example are respectively presented below,

$$
\begin{gathered}
\text { Mean field: }\left\{\begin{array}{l}
\frac{d}{d t} Y=\frac{1}{\tau_{c}}[b(1-\bar{u})+z] Y+\frac{\tilde{\gamma}}{\tau_{c}} Y^{3} \\
\frac{d \bar{u}}{d t}=\frac{1}{\tau_{I}}(1-\bar{u})-\frac{1}{\tau_{I}} \bar{u} e^{-\beta J_{0} \bar{u}+\beta h(Y)}
\end{array}\right. \\
\text { Stochastic averaging: } \frac{d}{d t} \bar{x}=\frac{1}{\tau_{c}}\left[b\left(1-u_{\beta, N}(h(\bar{x}))\right)+z\right] \bar{x}+\frac{\tilde{\gamma}}{\tau_{c}} \bar{x}^{3} .
\end{gathered}
$$

Although both the stochastic and mean-field closures are provided above for this example it is only the mean-field system which is expected to be valid for the parameter regimes of the numerical example in Figure 4.2. The stochastic closure (4.3) would only be valid if $\tau>>1$. The mean field closure (4.2) predicts nodes at $Y=0$ and $Y= \pm .1$. We display the solutions for the bistable deterministic/stochastic coupled system versus the mean-field equation in Figure 4.2. In general we note that: (a) The deterministic closure attains and remains for all time at the closest, according to dynamics, stable node based on its initial position. (b) Due to the high noise the stochastic process jumps occur almost immediately for the coupled system. The solution switches between the stable nodes of (4.2). Based on these findings it is clear that the deterministic closures will fail to capture the transient dynamics which are driven by stochasticity as Figure 4.2 strongly suggests, although they capture the correct average states. Similar failures are recorded, but not presented here, in the case of the stochastic averaging closure although since $\tau=.001$ this behavior is expected. The case of $\tau=.001<<1$ exhibits high noise values thus deterministic closures are certainly expected to fail. In the next section we examine how failure of the deterministic closures may still occur even though noise levels are not as high.

4.2. Example 2: Saddle Bifurcation. Strong noise is just one of many reasons for which the closures we presented so far may fail. What if strength of noise is in fact lowered? Could we have solutions which can still not be captured by our 

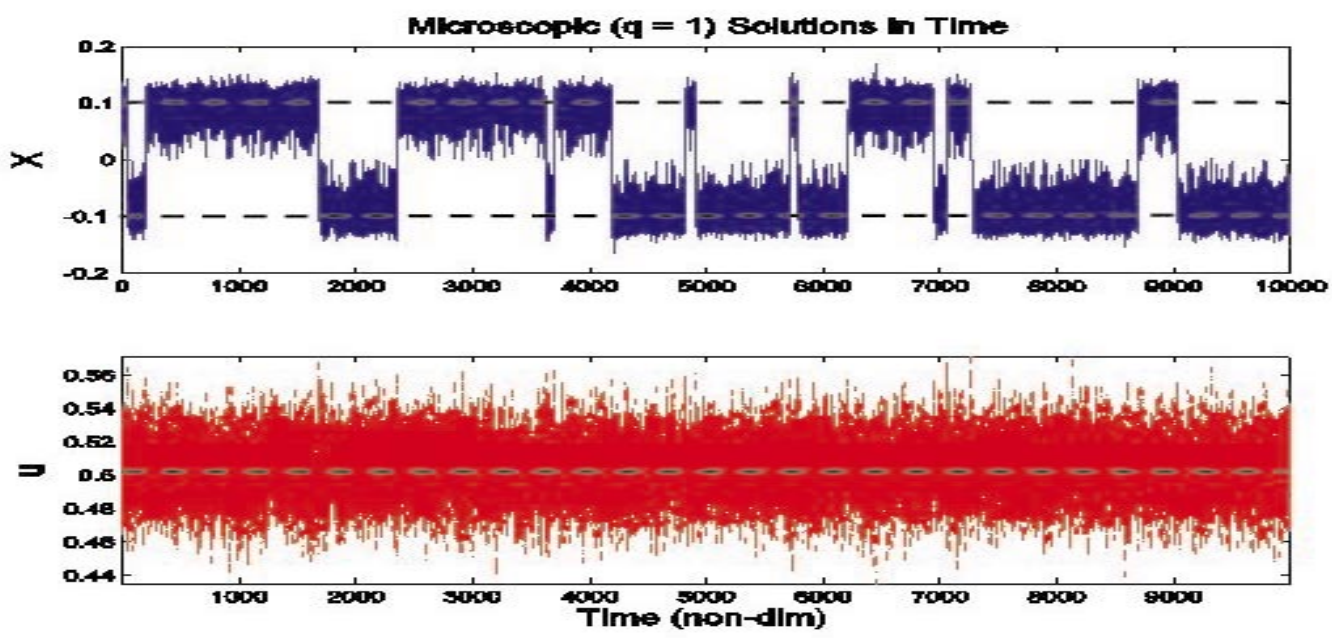

FIG. 4.2. Comparisons for the microscopic $(q=1)$ and mean-field solutions for the coupled system in the (4.1) case. Solutions presented for $\beta J_{0}=.01$ and $\tau=.001$ so that the noise levels are quite high. Other parameters: $L=20, N=1000$ with $b=4, z=-1, \tilde{\gamma}=100, c=-1$ and $h_{0}=.005$.

closures? We display below such an example where the failure in obtaining the correct system solution is not just attributed to noise but mainly to the occurrence of a rare event which can possibly appear in long enough time when the noise level is low; on the other hand, recall that the deterministic closures derived in Section 3 are valid only for finite times.

The saddle bifurcation example which we examine here has two fixed points which either appear or disappear based on the sign of the stochastic noise parameter $\bar{\sigma}$ which is expressed through the coupling. The specific dynamical system corresponding to $(2.1)$ is,

$$
\frac{d X}{d t}=\frac{1}{\tau_{c}}\left(r(\bar{\sigma})+\tilde{\gamma} X^{2}\right), \text { where we let } r(\bar{\sigma})=b(z-\bar{\sigma})
$$

We apply the following type of external potential: $h(X)=c X+h_{0}$. As usual all parameters are provided at the tables which follow. Here $b=1, z=.5, \tilde{\gamma}=-.05, c=$ $5, h_{0}=-1, \tau_{I}=1$ and $\beta J_{0}=.01$.

The ODE in (4.4) has a stability profile which depends on the sign of $r(\bar{\sigma})$. In the case of $r(\bar{\sigma})<0$ we obtain one stable and one unstable node at: $\pm \sqrt{-r(\bar{\sigma})) / \tilde{\gamma}}$. If however $r(\bar{\sigma})>0$ then no nodes exist. Last as $r(\bar{\sigma})$ approaches 0 then the two nodes at $\pm \sqrt{-r(\bar{\sigma})) / \tilde{\gamma}}$ merge and eventually disappear creating a saddle bifurcation. In this example we will set $\tau=1$. Although this value is not in the range of $\tau>>$ 1 the stochastic averaging closure could still be applied successfully based on our experimental simulation findings in [8]. We have similar expectations for the mean field closure. In that respect we present and briefly study both deterministic closures 

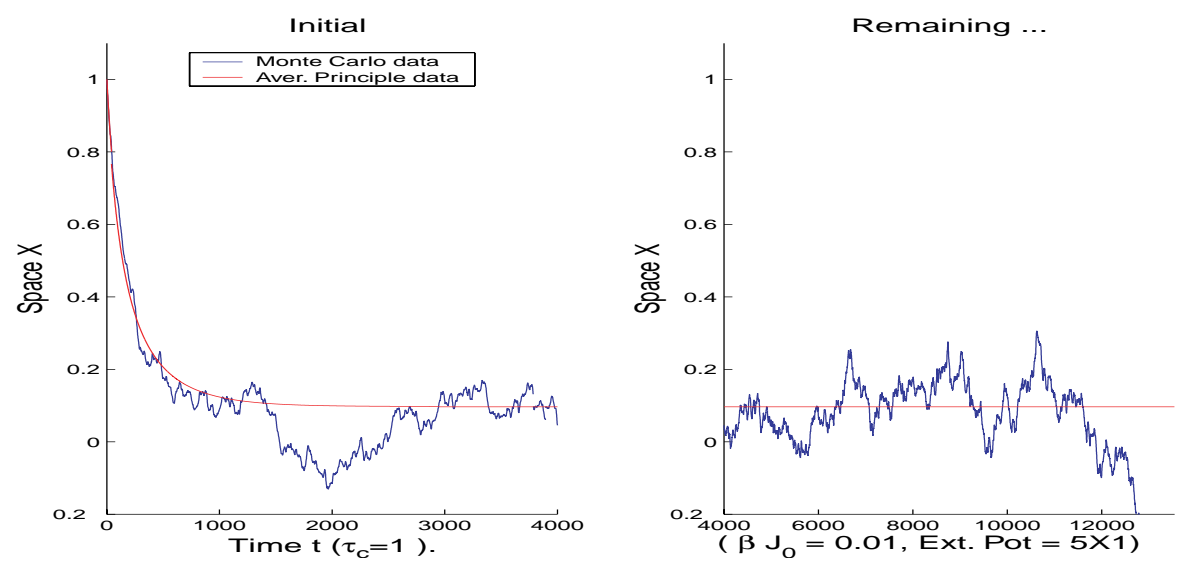

FIG. 4.3. Saddle ODE example. Extended time runs. Solutions for both microscopic Monte Carlo system and stochastic averaging closure. Parameters: $b=1, \tilde{\gamma}=-.05, \tau=1$ and $\beta J_{0}=.01$. Note the rare event jump which eventually drives the system to a finite time blow-up. The stochastic averaging closure is unable to track such a jump.

below

$$
\begin{gathered}
\text { Mean field: }\left\{\begin{aligned}
\frac{d}{d t} Y & =\frac{b}{\tau_{c}}[z-\bar{u}]+\frac{\tilde{\gamma}}{\tau_{c}} Y^{2} \\
\frac{d}{d t} \bar{u} & =\frac{1}{\tau_{I}}(1-\bar{u})-\frac{1}{\tau_{I}} \bar{u} e^{-\beta J_{0}} \bar{u}+\beta h(Y)
\end{aligned}\right. \\
\text { Stochastic averaging: } \quad \frac{d}{d t} \bar{x}=\frac{b}{\tau_{c}}\left[z-u_{\beta, N}(h(\bar{x})]+\frac{\tilde{\gamma}}{\tau_{c}} \bar{x}^{2} .\right.
\end{gathered}
$$

The solutions for both the averaging principle closure and the microscopic coupled system are displayed in Figure 4.3. A simple numerical study of both the mean field and stochastic averaging above shows that both predict the exact same stable node at $\bar{u} \approx .498, Y \approx .197$. This estimate is in complete agreement with the microscopic simulation of our hybrid system as can be seen in Figure 4.3. However both of these closures (4.5) and (4.6) are assumed to hold in finite time and this in fact is supported by our simulations.

Indeed the values predicted by the stochastic averaging closure are captured in the hybrid simulation as can be seen in the first part of Figure 4.3 for short times. However for long enough time a rare event jump occurs in the microscopic hybrid system which is not followed by the stochastic averaging or the mean field solution and creates a blow-up at finite times as Figure 4.3 suggests.

4.3. Example 3: Hopf Bifurcation. For our last major example in this subsection we examine the hybrid system $(2.1,2.2)$ where the ODE is of CGL type (2.12):

$$
\frac{d \vec{X}}{d t}=\frac{1}{\tau_{c}}\left[A \vec{X}-|\vec{X}|^{2} \vec{X}\right]
$$

where $A=\left(\begin{array}{cc}a(\bar{\sigma})+\gamma & -\omega \\ \omega & a(\bar{\sigma})-\gamma\end{array}\right),|\vec{X}|^{2}=X^{2}+Y^{2}$ and $a(\bar{\sigma})=b(\bar{\sigma}-z)$ with $b=4$ and $z=$ .5. We apply a linear external potential $h(\vec{X})=c\left(X+h_{0}\right)$ with $c=.5, h_{0}=-1$ and choose the remaining parameters as follows: $\omega=1, \tau_{I}=1, \tau_{c}=.01, \gamma=.9, \beta=.01, J_{0}=$ 
$1, X(0)=1, Y(0)=1$. Equation $(4.7)$ has a stable node at $(X, Y)=(0,0)$ for $a(\bar{\sigma})<0$. Otherwise if $a(\bar{\sigma}) \geq 0$ we have a limit cycle with radius depending on the size of $\bar{\sigma}$ (non-symmetric limit cycles occur for values of $\bar{\sigma}$ approaching 1 ).

The mean-field equations (3.7) for this hybrid model are

$$
\text { Mean field: }\left\{\begin{aligned}
\frac{d}{d t} Y_{1} & =\frac{1}{\tau_{c}}\left[(a(\bar{u})+\gamma) Y_{1}-\omega Y_{2}-Y_{1}^{3}-Y_{1}^{2} Y_{2}\right] \\
\frac{d}{d t} Y_{2} & =\frac{1}{\tau_{c}}\left[\omega Y_{1}+(a(\bar{u})-\gamma) Y_{2}-Y_{1}^{2} Y_{2}-Y_{2}^{3}\right] \\
\frac{d \bar{u}}{d t} & =\frac{1}{\tau_{I}}\left[1-\bar{u}-\bar{u} e^{-\beta J_{0} \bar{u}+\beta h(\vec{Y})}\right],
\end{aligned}\right.
$$

while the corresponding averaging closure for this case is,

$$
\text { Stochastic Averaging: }\left\{\begin{aligned}
\frac{d}{d t} \bar{x}_{t} & =\frac{1}{\tau_{c}}\left[\left(a\left(u_{\beta, N}\left(h\left(\bar{x}_{t}\right)\right)\right)+\gamma\right) \bar{x}_{t}-\omega \bar{y}_{t}-\bar{x}_{t}^{3}-\bar{x}_{t}^{2} \bar{y}_{t}\right] \\
\frac{d}{d t} \bar{y}_{t} & =\frac{1}{\tau_{c}}\left[\omega \bar{x}_{t}+\left(a\left(u_{\beta, N}\left(h\left(\bar{x}_{t}\right)\right)\right)-\gamma\right) \bar{y}_{t}-\bar{x}_{t}^{2} \bar{y}_{t}-\bar{y}_{t}^{3}\right] .
\end{aligned}\right.
$$
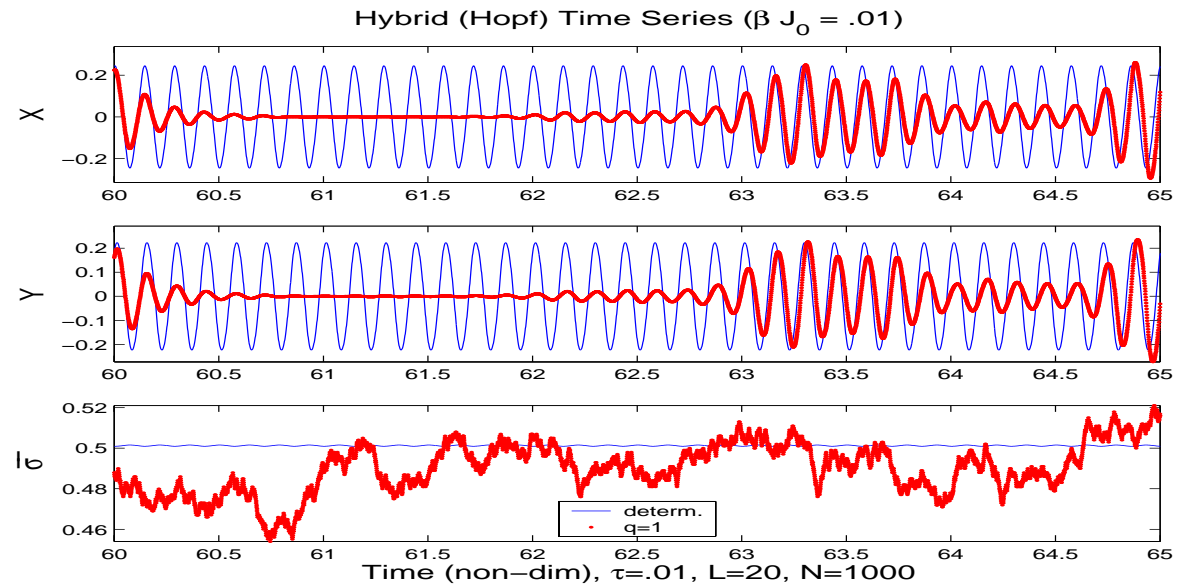

FIG. 4.4. CGL case. The mean field and microscopic hybrid system solutions compared in time. The mean field closure is unable to predict the interchange between the node at $\left(Y_{1}, Y_{2}, \bar{u}\right)$ and random radii limit cycles for this hybrid system. Parameters: $\tau=.01$ and $\beta J_{0}=.01$.

The stochastic averaging closure (4.9) is unable to track the correct behavior for the solutions of the system $(2.1,2.2)$ since the assumption $\tau>>1$ is violated for the parameter regimes of the numerical example in Figure 4.4. On the other hand the mean field closure (4.8) appears to be more suitable since no timescale separation assumption is required. The mean field closure however reveals constant radius limit cycles centered at $\left(Y_{1}, Y_{2}, \bar{u}\right) \approx(0,0, .5)$; this is also validated from analysis of the Jacobian corresponding to the linearization of (4.8) around such a state, which shows the following eigenvalues $\lambda_{Y_{1}} \approx .05+.41 i, \lambda_{Y_{2}} \approx .05-.41 i$ and $\lambda_{\bar{u}} \approx-2.1$. However, mean field fails to capture the true behavior of our hybrid system which consists of random interchanges between the node at $(0,0, .5)$ and limit cycles of random radii, see Figure 4.4; this phenomenon is clearly driven by noise which induces transitions through the bifurcation point defined by the sign of $a(\bar{\sigma})$.

\section{Coarse-Graining and Stochastic Closures}

Motivated by the examples in the previous section where stochastic noise played a crucial role in transient and long-time dynamics we now pursue the derivation of reduced stochastic approximations of the hybrid models that will allow both enhanced 
computational efficiency but also improved accuracy and predictive capability over deterministic closures. More precisely we obtain stochastic closures of the hybrid system in terms of a coupled ODE / coarse grained stochastic lattice model. Uncoupled stochastic coarse grained closures were first introduced in [9] and [10]. Here we extend these methods to hybrid systems and test their validity in the context of the three examples, presented earlier, where deterministic closures fail.

5.1. Coarse Graining of the Hybrid System. Using the empirical measure $\mu_{N}$ defined in (3.3) as a starting point, we define the coarse grained random process,

$$
\eta(k)=\mathcal{T}(\sigma)(k):=\sum_{x \in D_{k}} \sigma(x), \text { for } k=1, \ldots, n,
$$

and $\eta=\eta(k): k \in \mathcal{L}_{c}=\{1, \ldots, m\}$, with $\eta(k) \in\{0,1, \ldots, q\}$ the coverage of the coarse cell $D_{k}$ for $1 \leq k \leq m$ and $N=(m q)$. Note that each $D_{k}$ consists of $q$ microcells and the order parameter $\eta_{k}$ counts the number of particles in each coarse cell $D_{k}$. Here the coarse lattice $\mathcal{L}_{c}$ is defined through,

$$
\mathcal{L}_{c}=\frac{1}{m} Z \cap[0,1] \text { where } \mathcal{L}_{c} \subset \mathcal{L} .
$$

We define the coarse configuration space $\mathcal{H}_{m, q}=\{0,1, \ldots, q\}^{\mathcal{L}_{c}}$. The projection operator $\mathcal{T}$ is defined implicitly by (5.1). For any test function $g \in L^{\infty}\left(\mathcal{H}_{m, q} ; R\right)$ we have,

$$
f(\sigma):=g(\mathcal{T}(\sigma))=g(\eta)
$$

where $f$ is a test function in $L^{\infty}(\Sigma ; R)$. Therefore $(2.2)$ with generator $(2.3)$ can be written as follows in terms of the coarse lattice $\mathcal{L}_{c}$,

$$
\frac{d}{d t} E f(\sigma)=E \sum_{x \in \mathcal{L}} c(x, \sigma)\left[f\left(\sigma^{x}\right)-f(\sigma)\right]=E \sum_{k \in \mathcal{L}_{c}} \sum_{x \in D_{k}} c(x, \sigma)\left[f\left(\sigma^{x}\right)-f(\sigma)\right]
$$

Note that for $x \in D_{k}$ we have,

$$
\mathcal{T}\left(\sigma^{x}\right)(k)=\sum_{y \in D_{k}} \sigma^{x}(y)=\left\{\begin{array}{l}
\eta(k)+1 \text { when } \sigma(x)=0 \\
\eta(k)-1 \text { when } \sigma(x)=1
\end{array}\right.
$$

Thus the right hand side of (5.3) can be expressed in terms of $g$ as,

$$
f\left(\sigma^{x}\right)-f(\sigma)=(1-\sigma(x))\left[g\left(\eta+\delta_{k}\right)-g(\eta)\right]+\sigma(x)\left[g\left(\eta-\delta_{k}\right)-g(\eta)\right]
$$

where $\delta_{k} \in \mathcal{H}_{m, q}$ denotes the configuration with a single particle at site $k \in \mathcal{L}_{c}$. Rearranging the summations accordingly in the microscopic generator $L$ in (5.3) for $\sigma$ and corresponding coarse state $\eta=\mathcal{T}(\sigma)$ with rates from $(2.6)$ we obtain,

$$
\frac{d}{d t} E g(\eta)=\frac{d}{d t} E f(\sigma)=E \sum_{k \in \mathcal{L}_{c}} c_{a}(k)\left[g\left(\eta+\delta_{k}\right)-g(\eta)\right]+c_{d}(k)\left[g\left(\eta-\delta_{k}\right)-g(\eta)\right]
$$

where $g \in L^{\infty}\left(\mathcal{H}_{m, q} ; R\right)$ is a test function and $c_{a}(k), c_{d}(k)$ denote the exactly coarse grained adsorption and desorption rates,

$$
c_{a}(k)=\sum_{x \in D_{k}} c(x, \sigma)(1-\sigma(x)), \quad c_{d}(k)=\sum_{x \in D_{k}} c(x, \sigma) \sigma(x) .
$$


Note that these rates depend on the microscopic configuration $\sigma$ and not on the coarse random variable $\mathcal{T}(\sigma)$. In order to derive a Markov process for the coarse variable $\eta=\mathcal{T}(\sigma)$ we need to express these rates in terms of $\eta$. Indeed for the adsorption we have,

$$
c_{a}(k)=\sum_{x \in D_{k}} c(x, \sigma)(1-\sigma(x))=\sum_{x \in D_{k}} d_{0}(1-\sigma(x))=d_{0}[q-\eta(k)]
$$

where $d_{0}=1 / \tau_{I}$ and $U_{0}$ denotes the energy associated with the surface binding at $x$, (which for simplicity we take $U_{0}=0$ ). Based on relation (5.5) we can therefore define the coarse grained adsorption rate

$$
c_{a}(k, \eta)=d_{0}[q-\eta(k)]
$$

and expresses the rate by which $\eta(k)$ is increased by 1 . We wish to obtain a similar such relation for the desorption $c_{d}(k)$. Since $U(x)=\bar{U}(k)+O(q /(2 L+1))$ (see [8]) we have

$$
\begin{aligned}
c_{d}(k) & =\sum_{x \in D_{k}} c(x, \sigma) \sigma(x)=d_{0} \sum_{x \in D_{k}} \sigma(x) e^{-\beta\left(U_{0}+U(x)\right)} \\
& =d_{0} \sum_{x \in D_{k}} \sigma(x) e^{-\beta\left(U_{0}+U(x)\right)}=e^{-\beta\left(U_{0}+\bar{U}(k)+O\left(\frac{q}{2 L+1}\right)\right)} \sum_{x \in D_{k}} d_{0} \sigma(x) \\
& =d_{0} \eta(k) e^{-\beta\left(U_{0}+\bar{U}(k)\right)} \exp \left[O\left(\frac{q}{2 L+1}\right)\right]
\end{aligned}
$$

where

$$
\bar{U}(k)=\sum_{\substack{l \in \mathcal{L}_{c} \\ l \neq k}} \bar{J}(k, l) \eta(l)+\bar{J}(0,0)(\eta(k)-1)-h(\vec{X}),
$$

and the coarse grained interaction potential $\bar{J}(k, l)$ is computed as average of pairwise interactions between microscopic spins on coarse cells $D_{k}$ and $D_{l}$,

$$
\bar{J}(l, k)=m^{2} \int_{D_{k}} \int_{D_{l}} J(r-s) d r d s .
$$

Disregarding the $\exp \left[O\left(\frac{q}{2 L+1}\right)\right]$ term in $(5.7)$ we propose the following approximate desorption coarse grained rate,

$$
c_{d}(k, \eta)=d_{0} \eta(k) e^{-\beta\left(U_{0}+\bar{U}(k)\right)} .
$$

Based on rates (5.9) and (5.6) we can now provide the coarse grained Markov process generator for $\eta$ based on (5.4),

$$
L_{c} g(\eta)=\sum_{k \in \mathcal{L}_{c}} c_{a}(k, \eta)\left[g\left(\eta+\delta_{k}\right)-g(\eta)\right]+c_{d}(k, \eta)\left[g\left(\eta-\delta_{k}\right)-g(\eta)\right]
$$

and the corresponding coarse grained dynamics

$$
\frac{d}{d t} E g(\eta)=E L_{c} g(\eta), \quad \text { where } g \in L^{\infty}\left(\mathcal{H}_{m, q} ; R\right)
$$

Thus the hybrid system $(2.1,2.2)$ is approximated by the stochastic closure,

$$
\left\{\begin{aligned}
\frac{d}{d t} \vec{X} & =\frac{1}{\tau_{c}} G(\vec{X}, \bar{\eta}) \\
\frac{d}{d t} E g(\eta) & =E L_{c} g(\eta)
\end{aligned}\right.
$$


where $\bar{\eta}=\bar{\sigma}$. Note that in fact for this approximation to work we only require that $2 L+1>>q$ and not that $N \rightarrow \infty$ or $\tau<<1$ as is the case for the deterministic closures in Section 3; furthermore as in most asymptotics the condition $q<<2 L+1$ will require a relatively modest $q /(2 L+1)$ ratio. We will present further numerical as well as theoretical results showing the capabilities and possible limitations of this stochastic coarse grained closure in Section 6 .

\section{Validity Regimes of Stochastic Closures}

In this section we examine the coarse grained closure presented above for both the uncoupled and coupled spin flip dynamics cases and present both theoretical estimates of its capabilities as well as numerical simulations and comparisons against the microscopic hybrid system. There are several interesting issues regarding the extent to which we may apply this coarse graining method in order to approximate a microscopic dynamics process. In the derivation presented in Section 5 we did not make use of any underlying assumptions such as $N \rightarrow \infty$ as in the derivation of the mean field models or an assumption $\tau<<1$ on time scale separation. On the other hand the approximation itself included an error (5.7) which depends on the fraction of coarse graining $q$ over the interaction radius $L$. The main questions which we undertake to answer in this section are: (a) general comparison and capabilities of the coarse graining closure for the hybrid system $(2.1,2.2)$ under different parameter regimes (b) how far can we extend the assumption $q<<L$ and maintain a reasonably accurate approximation. We examine these and other similar questions below in the setting of our prototype hybrid system $(2.1,2.2)$ coupled to the ODE cases presented in Section 4 for which the deterministic closures failed.

6.1. Uncoupled spin flip dynamics. We begin with a theoretical estimate for the uncoupled dynamics case that gives a first concrete indication regarding the regimes of validity of the method.

First we define the concept of relative entropy that will provide a measure of accuracy for the proposed coarse grained procedure. We consider two probability measures $\pi_{1}(\sigma)$ and $\pi_{2}(\sigma)$ on the discrete state space $\mathcal{S}$. We further define the relative entropy for these measures as

$$
\mathcal{R}\left(\pi_{1} \mid \pi_{2}\right)=\sum_{\sigma \in \mathcal{S}} \pi_{1}(\sigma) \log \frac{\pi_{1}(\sigma)}{\pi_{2}(\sigma)} .
$$

Using Jensen's inequality we can show that the relative entropy can be thought of as a distance between two measures $\pi_{1}$ and $\pi_{2}$ since the following is true,

$$
\begin{aligned}
& \mathcal{R}\left(\pi_{1} \mid \pi_{2}\right) \geq 0 \text { and } \\
& \mathcal{R}\left(\pi_{1} \mid \pi_{2}\right)=0 \text { if and only if } \pi_{1}(\sigma)=\pi_{2}(\sigma) \text { for all } \sigma \in \mathcal{S} .
\end{aligned}
$$

Note that the relative entropy is not a true metric since $\mathcal{R}\left(\pi_{1} \mid \pi_{2}\right) \neq \mathcal{R}\left(\pi_{2} \mid \pi_{1}\right)$ for all possible measures $\pi_{1}, \pi_{2}$. Nevertheless there is an important inequality which allows us to use the relative entropy as a tool for estimating distance between two measures [22],

$$
\mathcal{R}\left(\pi_{1} \mid \pi_{2}\right) \geq C\left(\sum_{\sigma \in \mathcal{S}}\left|\pi_{1}(\sigma)-\pi_{2}(\sigma)\right|\right)^{2} \equiv C\left\|\pi_{1}-\pi_{2}\right\|_{1}^{2},
$$

where $C$ is an order one constant which is independent of the measures and the measure space. In order to compare the process $\{\mathcal{I} \sigma\}_{t \geq 0}$ and $\{\eta\}_{t \geq 0}$ we need to 
carry out an a priori analysis on the coarse path space $\mathcal{D}\left(\Sigma^{c}\right)$ i.e. on the space of all right-continuous paths $\eta_{t}:[0, \infty] \rightarrow \Sigma^{c}$. We denote by $Q_{\sigma_{0},[0, T]}$ the measure on $\mathcal{D}(\Sigma)$ for the process on the interval $[0, T],\left\{\sigma_{t}\right\}_{t \in[0, T]}$ with the initial distribution $\sigma_{0}$. Similarly $Q_{\eta_{0},[0, T]}^{c}$ denotes the measure on the coarse path space $\mathcal{D}\left(\Sigma^{c}\right)$. With a slight abuse of notation we also use $\mathcal{T}_{*} Q$ to denote the projection of the measure $Q$ on the coarse path space, i.e., the exact coarsening of the measure $Q$. Based on these definitions we have,

THEOREM 6.1. Suppose the process $\left\{\eta_{t}\right\}_{t \in[0, T]}$ defined by the coarse generator $\overline{\mathcal{L}}^{c}$ is the coarse approximation of the microscopic process $\left\{\sigma_{t}\right\}_{t \in[0, T]}$ then for any $q<L$ and $N$ where $m q=N$ the information loss as $q / L \rightarrow 0$ is

$$
\frac{1}{N} \mathcal{R}\left(\mathcal{T}_{*} Q_{\mathcal{T}_{*} \sigma_{0},[0, T]} \mid Q_{\eta_{0},[0, T]}^{c}\right)=T O\left(\frac{q}{2 L+1}\right) .
$$

We refer to [12] for the proof. The relative entropy estimate above demonstrates the limitations of the coarse graining method since it gives an order one error for nearest neighbor interactions $(L=1)$. This is not surprising in view of well-known renormalization calculations for the Ising model, as well as explicit numerical comparisons [9]. On the other hand, (5.7) rigorously identifies a small parameter in the coarse-graining process (see [9] for the analysis details), namely the ratio $q /(2 L+1)$; as it is the case with most asymptotics, from a practical/computational point of view a small parameter can be selected to be fairly large, see for instance the values of $q$ and $L$ in the simulations here and in $[9,11])$.

Although the estimate in Theorem 6.1 is for finite times $[0, T]$ only, and grows with $T$, it is still useful; in the case of phase transitions in the microscopic lattice the estimate ensures numerical accuracy during nucleation, which is typically an initial stage of the evolution. We refer to simulations partly motivated by our rigorous results in [11] that make precisely this point. In addition we have that, the coarsegrained Gibbs measure $\mu_{m, q}^{c g}$ lies within a controlled error from the exact coarse grained measure [13]. More specifically, we easily obtain the equilibrium version of Theorem 6.1 , namely

$$
\frac{1}{N} \mathcal{R}\left(\left(\mathcal{T}_{*} \mu^{N}\right) \mid \mu_{m, q}^{c g}\right)=O\left(\frac{q}{2 L+1}\right) .
$$

As a result of Theorem 6.1 and the Gibbs measure estimate (6.2), the transient, as well as the long time dynamics are expected to be captured accurately by the coarse grained closure. Indeed, we display numerical comparisons in Figure 6.1 of the coarse graining closure against the corresponding microscopic dynamics for the uncoupled equation (2.2) under three cases of dynamics: attractive $\left(\beta J_{0}=2\right)$, repulsive $\left(\beta J_{0}=-4\right)$ and non-interacting $\left(\beta J_{0}=0\right)$. In the simulations presented in Figure 6.1 we test pathwise agreement toward equilibrium between both the microscopic as well as the coarse grained process but also the level and effects of noise. For these simulations we apply a piecewise constant potential with $L=100$ while the number of particles is $N=1000$. We used the same seed for the random number generator so that we will be able to compare effects attributed to each solution and not to different paths of the stochastic process itself. For further details on the numerics we refer to the Appendix Section A.

Based on the simulations in Figure 6.1 the coarse graining closure displays remarkable agreement with respect to the microscopic process. The coarse grained closure 


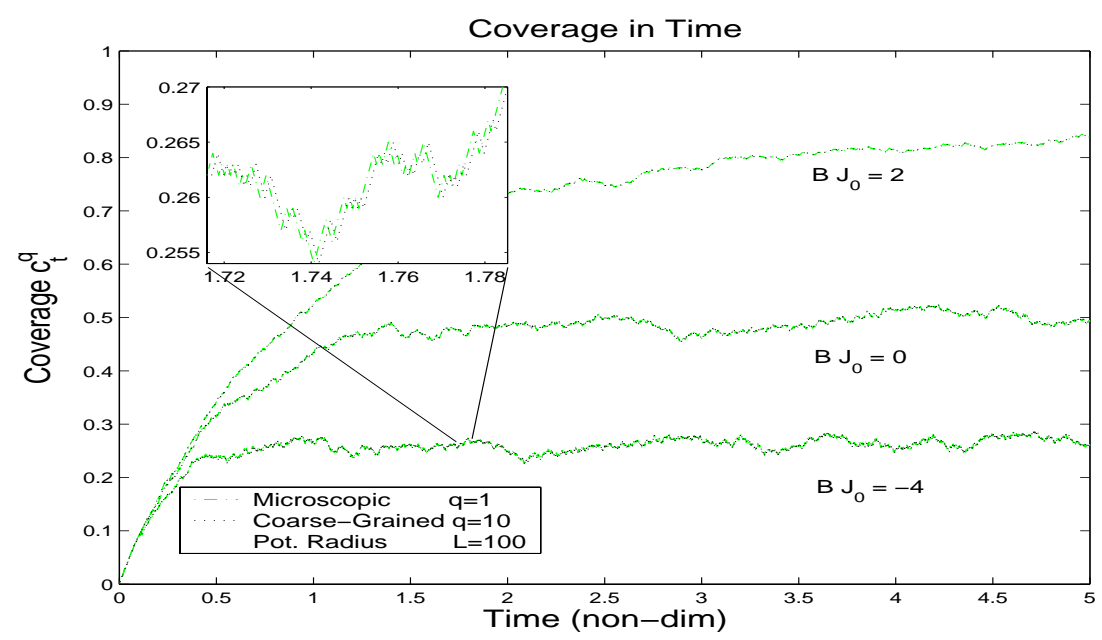

FIG. 6.1. Microscopic versus coarse grained $(q=10)$ relaxation dynamics for the uncoupled stochastic model (2.2) under three different dynamics (attractive) $\beta J_{0}=2$, (non-interacting) $\beta J_{0}=0$ and (repulsive) $\beta J_{0}=-4$. The coarse grained process seems to both, follow the microscopic dynamics to the correct equilibrium dynamics but also capture correctly the noise on its transition path towards equilibrium. We zoom in the case $\beta J_{0}=-4$ in order to further display the agreement between the two processes. Other parameters: $L=100, N=1000$

presented in this figure tracks well not only the path to equilibrium but also the respective noise in time. However this is not entirely surprising due to the estimate in Theorem 6.1 as well as (6.2).

6.2. Stochastic Fluctuations in Hybrid Systems. In the following subsections we present numerical examples of the coarse graining closure for a number of different types of coupled systems. We examine couplings with ODEs of the type considered in (2.10-2.12). Although a large number of numerical test cases were undertaken only a limited representative number are shown here through tables and figures while our general findings are summarized. We give particular emphasis on the parameter regimes for which the deterministic closures were not able to produce accurate approximations and specifically revisit the related examples from Section 4 . Note that we focus on numerical examples with interaction potentials of different sizes $L$ but not $L=1$ (nearest neighbor) since our theoretical results imply more accuracy as $L \rightarrow N$ and $q \rightarrow 1$.

6.2.1. Example 1: Bistable Case. Here we re-examine Example 1 from Section 4.1 using the exact same parameters. We carried out comparisons of the microscopic and coarse grained hybrid system solutions for $X$ and $\bar{\sigma}$ corresponding to $\tau=5,1$ and .1 respectively for the case of $\beta J_{0}=.01$ where typically the noise levels are highest.

In the following table we provide further simulation results in terms of CPU 


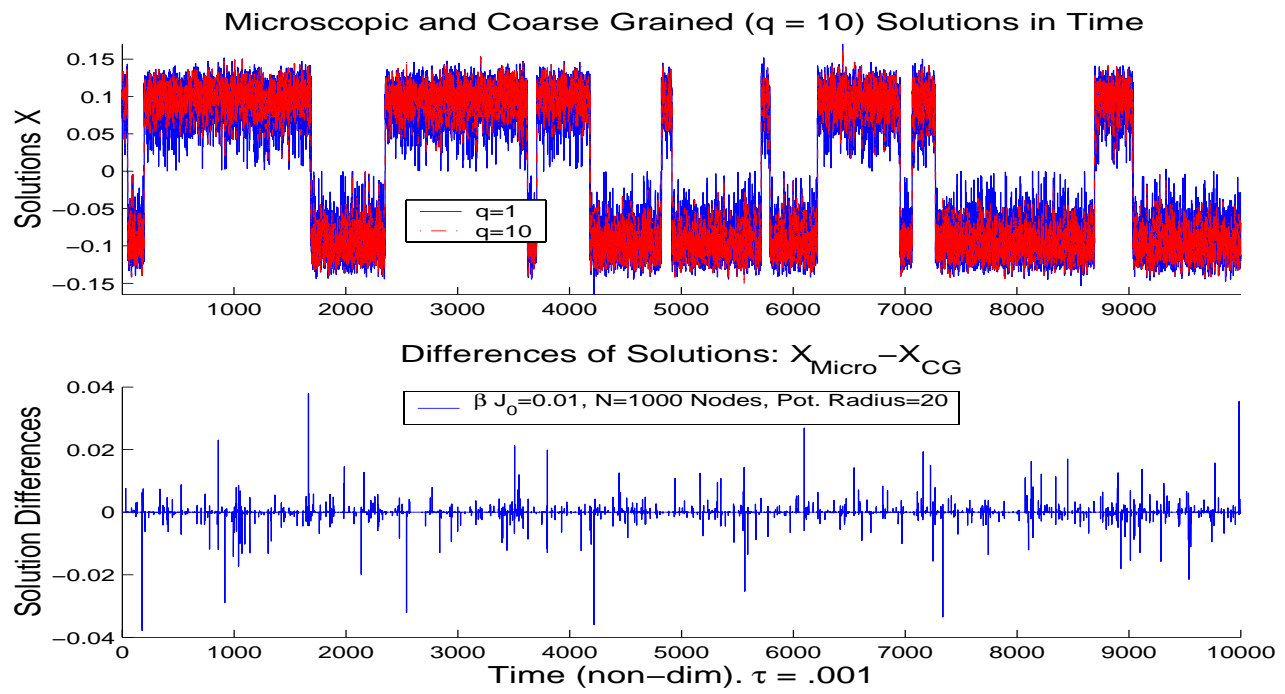

FIG. 6.2. Pathwise comparisons of system (2.1,2.2) for the bistable bifurcation ODE (4.1). We observe complete agreement for the cases presented here of the microscopic and coarse grained $(q=10)$ simulations. Solutions presented for the case of $\beta J_{0}=.01$ and $\tau=.001$ so that the noise levels are quite high. Other parameters: $b=4, z=-1, \tilde{\gamma}=100, c=1$ and $h_{0}=.005$.

comparisons for the hybrid system above:

\begin{tabular}{|l||c|}
\hline System Type & CPU Time \\
\hline \hline Microscopic & $6695 \mathrm{sec}$. \\
Coarse Grained at $q=10$ & $237 \mathrm{sec}$. \\
Coarse Grained at $q=20$ & $93 \mathrm{sec}$. \\
\hline Coarse Grained at $q=50$ & $44 \mathrm{sec}$. \\
Coarse Grained at $q=100$ & $28 \mathrm{sec}$. \\
\hline
\end{tabular}

Note the CPU reduction even for small $q$ values. The horizontal line in the table separating the cases $q=20$ and $q=50$ denotes the point after which we seem to have coarse grained excessively. This is once again in agreement with our theoretical estimate in Theorem 6.1. In fact the cases of $q=50$ and 100 examined numerically in the table above showed progressive deterioration in agreement between the coarse grained and microscopic dynamics. The simulations corresponding to the cases of $q=10$ and $q=100$ are presented in Figures 6.2 and 6.3 respectively. The case of $q=10$ shows excellent agreement between the two dynamics while the case for $q=100$ displays the expected deterioration.

6.2.2. Example 2: Saddle Bifurcation. We now re-examine Example 2 from Section 4.2. We will show that the solutions display pathwise agreement between the microscopic and coarse grained Monte Carlo simulation for long time. Note that for this example the stability profile changes every time the stochastic process parameter $\bar{\sigma}$ passes through .5 . We run this simulation far enough in time until the rare event which we witnessed before in Section 4 occurs. As expected the rare event jump is leading to finite time blow-up. Remarkably the random jumps for both the microscopic and coarse grained closures occur at almost the same locations. 


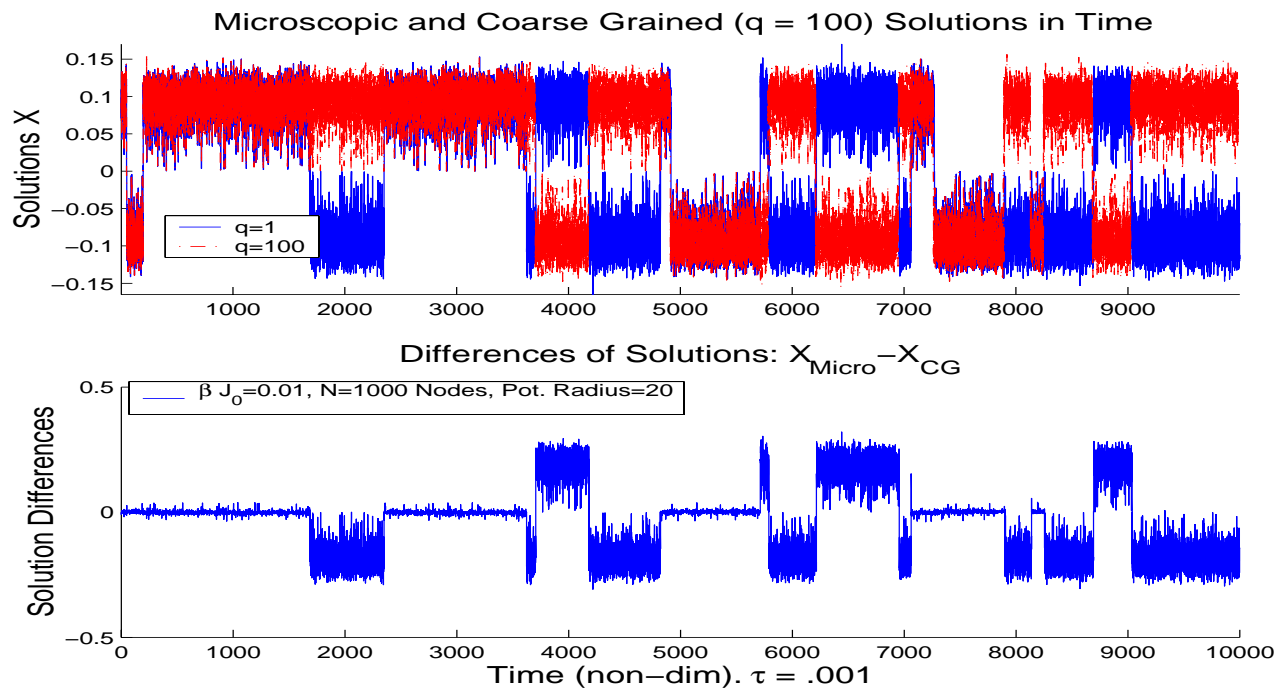

FIG. 6.3. An example of over-coarse graining according to Theorem 6.1. Pathwise comparisons of the same system (2.1,2.2) with the bistable bifurcation ODE (4.1). We do not observe as much agreement for the cases presented here between the microscopic and coarse grained $(q=100)$ simulations. Solutions presented for the case of $\beta J_{0}=.01$ and $\tau=.001$ so that the noise levels are quite high. Other parameters: $b=4, z=-1, \tilde{\gamma}=100, c=1$ and $h_{0}=.005$.

We compare the solutions of this coupled system in Figure 6.4 for three different types of coarse grainings: Microscopic $(q=1)$, coarse grained with $q=10,20$. Note that all three simulations fall almost on top of each other with small discrepancies at the very moment that we observe the finite-time blow up.

We summarize the comparisons for the microscopic Monte Carlo simulations for this example against the equivalent coarse grained and averaging principle solutions of (5.12) in the table below. The averaging principle statistics are also included from [8] for comparison purposes. Note the remarkable agreement regardless of relaxation time $\tau$ or value of $\beta J_{0}$ ! All numerics run up to a time of $t=1000$ (or 10000 data samples). 


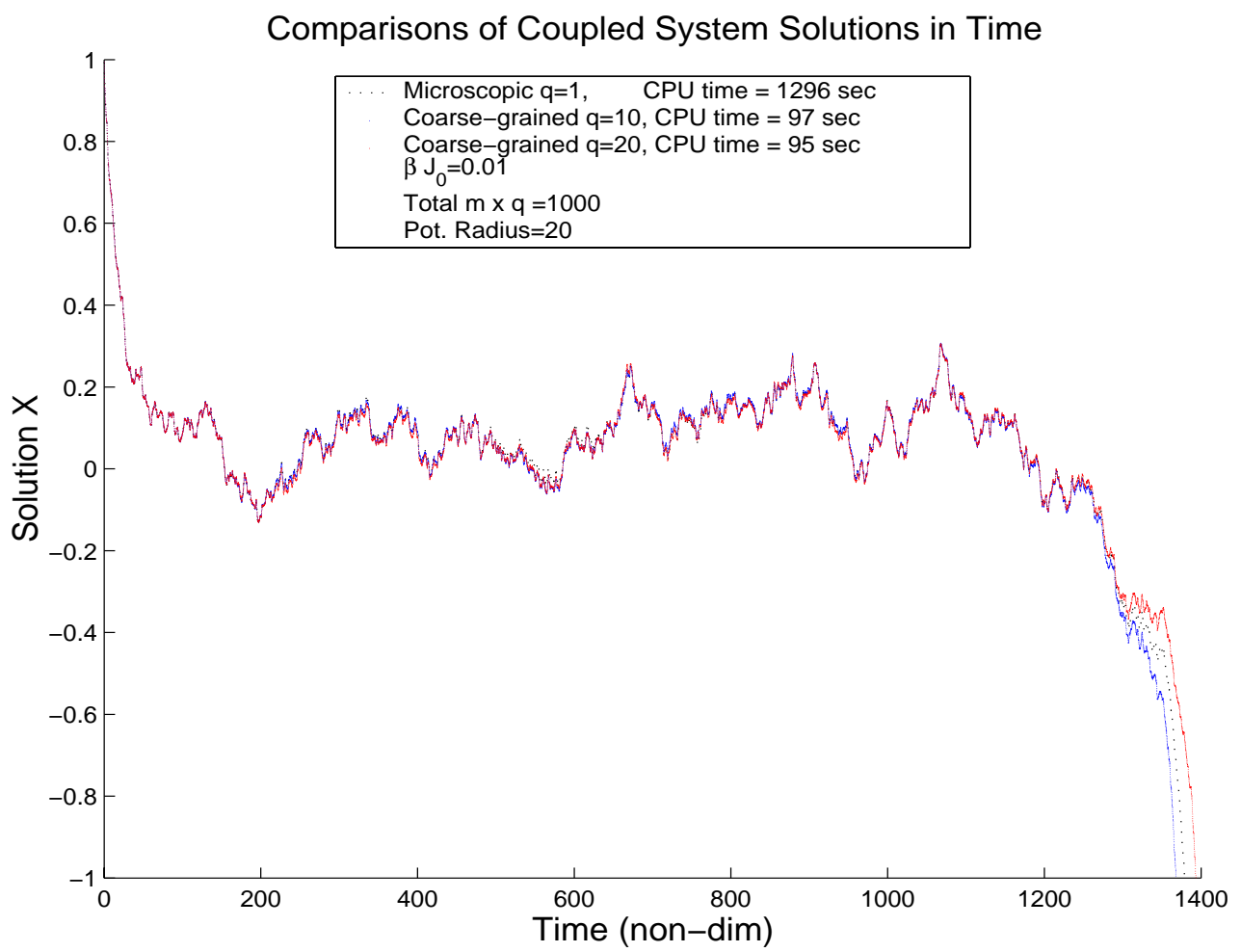

FIG. 6.4. Example of saddle ODE achieving a rare event potential well escape solution for the coupled system. Long time statistics for the case of $\beta J_{0}=.01$ and $\tau=1$. Other parameters: $b=1 ., \tilde{\gamma}=-.05$

Relative Error Comparisons: $\left|\bar{X}_{M i c}-\bar{X}_{A P}\right| /\left|\bar{X}_{A P}\right|$.

Microscopic vs Averaging Principle

\begin{tabular}{lccc} 
Case & $\beta J_{0}=.01$ & $\beta J_{0}=2$ & $\beta J_{0}=-2$ \\
\hline$\tau=.1$ & $\infty$ & .1 & .71 \\
$\tau=1$ & .87 & .04 & 0. \\
$\tau=5$ & .08 & .04 & 0.
\end{tabular}

Relative Error Comparisons: $\left|\bar{X}_{M i c}-\bar{X}_{C G}\right| /\left|\bar{X}_{C G}\right|$.

Microscopic vs Coarse Grained

\begin{tabular}{lccc} 
Case & $\beta J_{0}=.01$ & $\beta J_{0}=2$ & $\beta J_{0}=-2$ \\
\hline$\tau=.1$ & - & 0. & 0. \\
$\tau=1$ & .05 & 0. & 0. \\
$\tau=5$ & 0. & 0. & 0.
\end{tabular}

In general we observe significant agreement between the microscopic and coarsegrained Monte Carlo solutions regardless of relaxation time used as well as significant speed up in CPU time (see Figure 6.4).

6.2.3. Example 3: Hopf bifurcation. For our last major example in this subsection we revisit Example 3 from Section 4.3 using the exact same parameters as in Section 4.3.

In Figure 6.5 we display the solutions from the mean field closure, the hybrid 
Hybrid (Hopf) Time Series $\left(\beta J_{0}=0.01\right)$
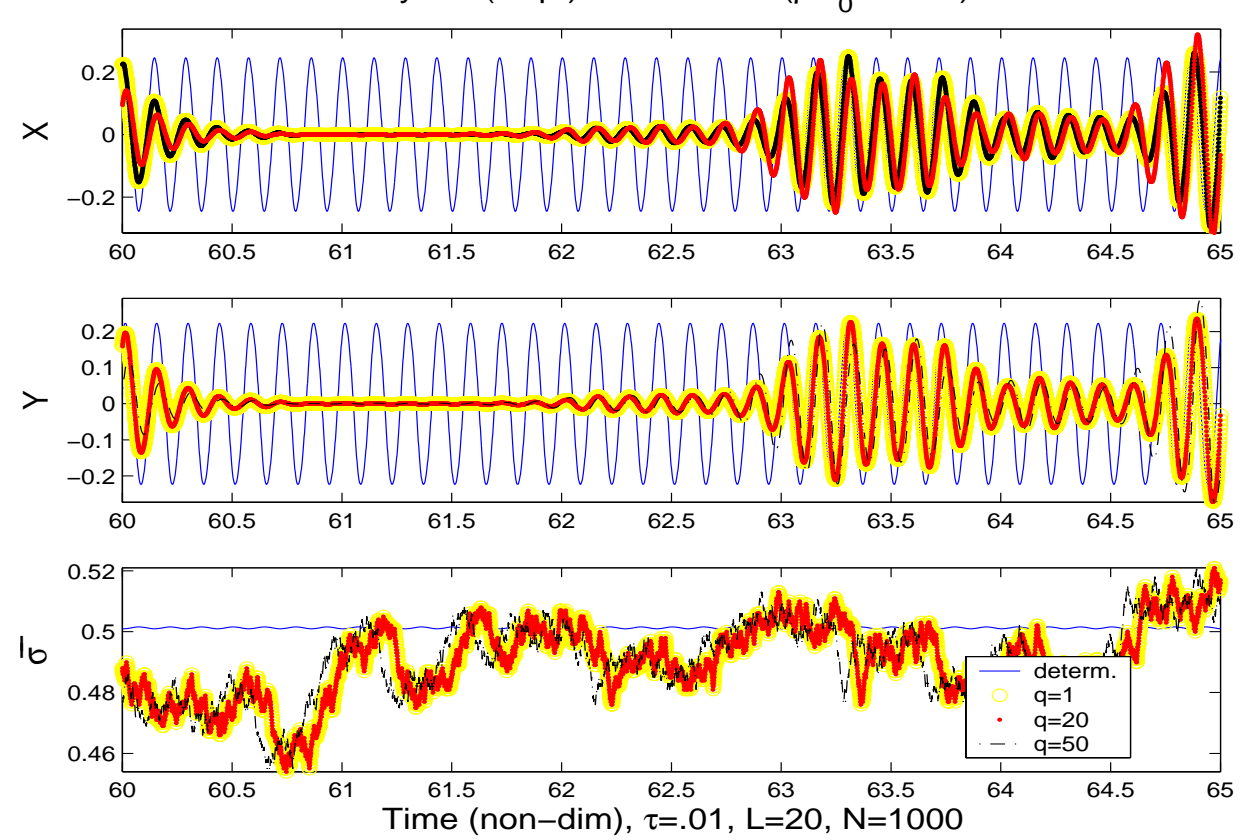

FIG. 6.5. Saddle ODE: Comparisons between the solutions of our hybrid system (5.12) and the mean field closure, microscopic (4.7), coarse grained closure for $q=20$ and (over) coarse grained closure for $q=50$. Same parameters as in Figure 4.4: $\tau=.001$ and $\beta J_{0}=.01$.

system solution $(q=1)$ and the coarse grained solutions for $q=20$ and $q=50$. We observe that once again the coarse grained closure captures correctly the behavior of our hybrid system. Furthermore we show numerical evidence of over-coarse graining in the case of $q=50$ where we observe a small deterioration in the approximation of the solution in accordance with the spirit of Theorem 6.1 .

Last we also present CPU comparisons for different coarse graining values for this example in the following table:

\begin{tabular}{|l||ccc|cc|}
\hline Coarse Graining & Microscopic & $\mathrm{q}=10$ & $\mathrm{q}=20$ & $\mathrm{q}=50$ & $\mathrm{q}=100$ \\
\hline \hline CPU Time & $10225 \mathrm{sec}$ & 451 sec & 201 sec & 106 sec & $95 \mathrm{sec}$ \\
\hline
\end{tabular}

The vertical line between $q=20$ and $q=50$ denotes that we have coarse grained too much, according to the results in Theorem 6.1. Surprisingly however, even though we over-coarse grained relative to $L=20$, the case $q=50$ seems to also approximate well the microscopic dynamics as can be seen in Figure 6.5.

REMARK 6.2. We can also carry out detailed statistical analysis including power spectra, auto- and cross-correlations for the microscopic and the coarse grained time series. However here the results are conclusive since we have demonstrated pathwise agreement between microscopic and coarse grained dynamics. On the other hand we refer to [7] where such a statistical analysis is undertaken for more challenging examples exhibiting intermittency and random oscillations in the presence of phase transitions in the microscopic dynamics. 


\section{Conclusions and discussion}

In this paper we studied the influence of micro- or sub- grid scale noise in hybrid dynamical systems consisting of coupled macroscopic, deterministic differential equations and microscopic, stochastic lattice particle systems. We focused on model prototype systems proposed in the earlier companion paper [8] that are computationally tractable with direct numerical simulation even for long times, but can still exhibit a host of complex phenomena, as further demonstrated here. Our findings strongly suggest that deterministic closures of the hybrid system such as mean field, stochastic averaging or moment equations may be either inadequate as descriptions of the overall system or simply difficult to both derive and assess in their effectiveness. Specifically, we have established that deterministic closures have substantial deviations from the hybrid system in both transient and long-time regimes: (a) high noise levels can account for failures in deterministic closures regardless of simulation time length and, (b) similarly weak noise becomes important in long times and induces rare events that can substantially alter the long-time dynamics.

Motivated by such considerations, we proposed a class of stochastic closures based on systematic coarse-graining of the microscopic stochastic lattice dynamics that yield computationally inexpensive reduced hybrid models and capture correctly the transient and long-time behavior of the full hybrid system, including rare events and random transitions between stable points and bifurcation regimes. In particular, based on the simulations our findings for the coarse grained closure are as follows: (a) Agreement with microscopic dynamics even if $\tau$ is not in the range of $>>1$ for very long times (b) Pathwise agreement (c) Agreement even in case of solutions with stable nodes which switch in time due to fluctuations. Those are solutions which due to the inherent stochastic noise in the system move between successive potential wells. Remarkably the coarse grained simulation follows these moves exactly for very long times (d) Remarkable CPU time improvement based on the size $q$ of coarse graining used. (e) Care must be taken so as to not over coarse grain since in that case we see a deterioration in accuracy of the coarse grained simulation. There is a correlation between the size of the potential radius $L$ and the size of coarse graining $q$ after which this deterioration starts to occur, which is mathematically quantified by the relative entropy estimates in Section 6.1. Similarly, based on Theorem 6.1, the nearest neighbor case of $L=1$ should produce higher approximation errors. Cases with smaller interaction potentials $(L=10)$ have also been examined successfully in [11] for uncoupled dynamics but the extent for which the approximation will succeed for small $L$ should be probed further.

In this paper we exclusively focused on hybrid systems where phase transitions do not occur in the microscopic lattice dynamics; on the other hand complex dynamic behavior was due to the bistability or the type of bifurcation behavior of the externally coupled deterministic system, and its coupling with the microscopic stochastic model. Phase transitions in the microscopic lattice system are due to strong particle/particle interactions and such hybrid systems demonstrate even more complex behavior than the ones considered here, for instance regimes of strong intermittency, and random oscillations; this case is treated separately in a follow-up publication [7].

\section{Appendix A. Numerical Implementations.}

The full coupled system is numerically solved by performing both, a Monte Carlo simulation for (2.2) and an adaptive ODE solver for (2.1). Each iteration of the Monte Carlo simulation produces a variable time step $\Delta t_{i}$ within which a spin-flip occurs at 
a specific lattice node based on the transition probability

$$
\left[c_{a}(k, \sigma)+c_{d}(k, \sigma)\right] \Delta t_{i}+O\left(\Delta t_{i}^{2}\right)
$$

where $k$ denotes a lattice location and spin $\sigma$. The Monte Carlo simulation proceeds by flipping spins, for a total given time $\Delta t=\sum_{i} \Delta t_{i}$ and then pauses. The ODE solver then iterates until the given time step $\Delta t$ has been exhausted. This procedure repeats until the total time is exhausted or other types of stopping criteria have been met.

Specifically we obtain a solution of (2.2) by implementing a process-type kinetic Monte Carlo (KMC) algorithm [2] for spin flip Arrhenius dynamics. The microscopic algorithm itself has been presented in [8] and we direct the interested reader there for the details. The coarse grained versions of the algorithm follows the exact same numerical implementation using the corresponding coarse grained rates from Section 5 .

As expected a kinetic Monte Carlo algorithm produces no "null" steps and therefore every iteration is a success. (A version of the pseudo-code just described has also been created which implements a local update scheme at every iteration thus improving speed at the reciprocal expense of allocating more computer memory).

To solve our ODEs we employ a 4th order adaptive Runge-Kutta-Fehlberg (RKF). The scheme applies a Runge-Kutta method of order 5 to estimate the error in a RungeKutta method of order 4. Further the scheme enforces a computational advantage of performing only six evaluations per time step instead of eleven. At each iteration, the time step is adjusted up or down to control the error.

We use a finite size interaction potential range $L<\infty$. In all simulations in this work we set $N=1000$ lattice nodes. We produce simulations and compare observables at microscopic $(q=1)$ and coarse-grained $(q>1)$ levels. For consistency purposes we use the same seeds for our random number generator in order to be able to compare simulations for different coarse grained values of $q$. This allows us to focus on the differences attributed only to the coarse graining variable and not on those resulting from different paths due to the initial seed. In the case of several realizations we initialize each new microscopic realization with a different seed. Once again for comparison purposes, we initialize each subsequent coarse grained realization with the same seed used in the respective microscopic simulations. All simulations are compared in the same non-dimensional time units.

Acknowledgments. The research of M.A.K. is partially supported by NSFDMS-0413864 and NSF-ITR-0219211, the research of A.J.M. is partially supported by ONR N00014-96-1-0043, NSF-DMS-9972865 and NSF-CMG-0222133.

\section{REFERENCES}

[1] R. S. Aranson and L. Kramer, The world of the complex Ginzburg-Landau equation, Reviews of Modern Physics, 74, 99-143, 2002.

[2] A. Bortz, M. Kalos and J. Lebowitz, A new algorithm for Monte Carlo simulation of Ising spin systems, J. Comput. Phys., 17, 10-18, 1975.

[3] A. Brandt, Multi-scale Computation: From Fast Solvers To Systematic Upscaling, Computational Fluid And Solid Mechanics, Elsevier, 2003.

[4] W. E and B. Engquist, The heterogeneous multiscale method, Comm. Math. Sci., 1, 87-133, 2003.

[5] R. Ellis, Entropy, Large Deviations, and Statistical Mechanics, Springer-Verlag, 1985.

[6] M. I. Friedlin and A. D. Wentzell, Random Perturbations of Dynamical Systems, Springer, 1998. 
[7] M. A. Katsoulakis, A. J. Majda and A. Sopasakis, Intermittency, Metastability and Coarse Graining for Coupled Deterministic-Stochastic Systems, in progress.

[8] M. A. Katsoulakis, A. J. Majda and A. Sopasakis, Multiscale couplings in prototype hybrid deterministic/stochastic systems: part I, deterministic closures, Comm. Math. Sci., 2, 255-294, 2004.

[9] M. A. Katsoulakis, A. J. Majda and D. G. Vlachos, Coarse-grained stochastic processes and Monte Carlo simulations in lattice systems, J. Comp. Phys., 186, 250-278, 2003.

[10] M. A. Katsoulakis, A. J. Majda and D. G. Vlachos, Coarse-grained stochastic processes for lattice systems, Proc. Natl. Acad. Sci., 100, 782-787, 2003.

[11] M. A. Katsoulakis, P. Plecháč and A. Sopasakis, Error control and analysis in coarsegraining of stochastic lattice dynamics, submitted, 2005.

[12] M. A. Katsoulakis and J. Trashorras, Information loss in coarse-graining of stochastic particle dynamics, J. Stat. Phys., submitted, 2004.

[13] M. A. Katsoulakis and D. G. Vlachos, Hierarchical kinetic Monte Carlo simulations for diffusion of interacting molecules, J. Chem. Phys., 112, 18, 2003.

[14] I. G. Kevrekidis, C. W. Gear, J. Hyman, P. G. Kevrekidis, O. Runborg and C. Theodoropoulos, Equation-free, coarse-grained multiscale computation: enabling microscopic simulators to perform system-level tasks, Comm. Math. Sci., 1, 715-762, 2003.

[15] B. Khouider, A. J. Majda and M. A. Katsoulakis, Coarse-grained stochastic models for tropical convection and climate, Proc. Natl. Acad. Sci., 100, 11941-11946, 2003.

[16] C. Kipnis and C. Landim, Scaling Limits of Interacting Particle Systems, Springer, 1999.

[17] R. Lam and D. G. Vlachos, Multiscale model for epitaxial growth of films: growth mode transition, Phys. Rev. B, 64, 35401, 2001.

[18] T. M. Liggett, Stochastic Interacting Systems: Contact Process, Voter and Exclusion Process, Springer-Verlag, Berlin Heidelberg, 1999.

[19] A. J. Majda and B. Khouider, Stochastic and mesoscopic models for tropical convection, Proc. Nat. Acad. Sci., 99, 1123, 2002.

[20] H. C. Oettinger and M. Laso, Simulating Polymer Flow, Simulation Methods for Modeling Polymers, 2004

[21] S. T. Rodgers and K. F. Jensen, Multiscale modeling of chemical vapor deposition, J. Appl. Phys., 83, 1, 524, 1998.

[22] T. M. Thomas and J. A. Cover, Elements of Information Theory, Wiley, 1991.

[23] D. G. Vlachos, L. D. Schmidt and R. Aris, The effects of phase transitions, surface diffusion and defects on surface catalyzed reactions: fluctuations and oscillations, J. Chem. Phys., 93, 8306, 1990.

[24] H. S. Wiley, S. Y. Shvartsman and D. A. Lauffenburger, Computational modeling of the EGF receptor system: a paradigm for systems biology, Trends Cell Biol., 13, 43-50, 2003. 